\title{
A GENERALIZATION OF SUSPENSION THEOREMS
}

\section{YASUTOSHI NOMURA}

Our purpose in this note is to establish a classification theorem for fiberings with a loop-space as fibre. This is deduced by applying a generalized suspension theorem which will be proved in $\S 1$. As a by-product we obtain a proposition concerning fiberings with a loop-space as the total. Throughout this note we shall denote by $\mathfrak{B}$ the category of spaces having the based homotopy type of a $C W$-complex.

\section{§ 1. Generalized suspension theorems}

For a given map $f: X \rightarrow Y$, let $E_{f}$ denote the subspace of $X \times E Y$ consisting of the pairs $(x, \beta)$ such that $f(x)=\beta(1)$ where $E Y$ is the space of paths in $Y$ emanating from the base-point $y_{0}$, and let $C_{f}$ denote the space obtained by attaching the reduced cone over $X$ to $Y$ by means of $f$. Denoting the loop and suspension functors respectively by $\Omega$ and $S$, we have defined in $[6, \mathrm{p}$. 136] $\eta^{\prime}: S E_{f} \rightarrow C_{f}$ and $\eta: E_{f} \rightarrow \Omega C_{f}$ by setting, for $(x, \beta) \in E_{f}$,

$$
\begin{aligned}
& \eta^{\prime}((x, \beta), s)= \begin{cases}\beta(2 s) & \text { if } 0 \leqq s \leqq \frac{1}{2}, \\
(x, 2-2 s) & \text { if } \frac{1}{2} \leqq s \leqq 1,\end{cases} \\
& \{\eta(x, \beta)\}(s)= \begin{cases}(x, 2 s) & \text { if } 0 \leqq s \leqq \frac{1}{2}, \\
\beta(2-2 s) & \text { if } \frac{1}{2} \leqq s \leqq 1 .\end{cases}
\end{aligned}
$$

These induce suspensions $\sigma^{*}=\left(\eta^{\prime}\right)^{*}: \pi\left(C_{f}, Z\right) \rightarrow \pi\left(S E_{f}, Z\right)$ and $\sigma_{*}=\eta_{*}: \pi\left(Z, E_{f}\right)$ $\rightarrow \pi\left(Z, \Omega C_{f}\right)$ for any space $Z$, where $\pi(A, B)$ denotes the set of homotopy classes of maps $A \rightarrow B$. The following has been established there:

THEOREM 1. If $Y$ is r-connected and $E_{f}$ s-connected, then $\sigma^{*}: H^{q}\left(C_{f}\right)$ $\rightarrow H^{q}\left(S E_{f}\right)$ is an isomorphism for $q \leqq r+s+1$ and a monomorphism for $q=r+s+2$.

Received April 26, 1961. 
TheOREM 1'. Let $X$ and $C_{f}$ be $r$-and s-connected respectively. Then $\sigma_{*}$ : $\pi_{q}\left(E_{f}\right) \rightarrow \pi_{q}\left(\Omega C_{f}\right)$ is an isomorphism for $1 \leqq q \leqq r+s-1$ and an epimorphism for $q=r+s$.

These theorems may be generalized as follows:

THEOREM 2. Let $X$ and $Y$ both belong to $\mathfrak{W}$, and let $Y$ and $E_{f}$ be $r$ - and s-connected respectively $(r \geqq 1, s \geqq 0$ ). Suppose that $Z$ is $n$-simple for all $n>0$. Then

(a) $\sigma^{*}: \pi\left(C_{f}, Z\right) \rightarrow \pi\left(S E_{f}, Z\right)$ is onto if $\pi_{q}(Z)=0$ for $q \geqq r+s+2$.

(b) $\sigma^{*}: \pi\left(C_{f}, Z\right) \rightarrow \pi\left(S E_{f}, Z\right)$ is $1-1$ if $\pi_{q}(Z)=0$ for $q \geqq r+s+3$.

Theorem $2^{\prime}$. Let $Z$ be in $\mathfrak{M}$ and let $X$ aud $C_{f}$ be $r$ - and s-connected respectively $(r \geqq 1, s \geqq 2)$. Then

(a) $\sigma_{*} ; \pi\left(Z, E_{f}\right) \rightarrow \pi\left(Z, \Omega C_{f}\right)$ is $1-1$ if the integral cohomology groups $H^{q}(Z)$ are trivial for $q \geqq r+s$.

(b) $\sigma_{*}: \pi\left(Z, E_{f}\right) \rightarrow \pi\left(Z, \Omega C_{f}\right)$ is onto if $H^{q}(Z)=0$ for $q \geqq r+s+1$.

The proofs will be based on the following simple:

Lemma. Let $X$ and $Y$ be in $\mathfrak{B}$ and let $f: X \rightarrow Y$ be a given map. Then (i) $f$ is equivalent to an inclusion $X^{\prime} \subset Y^{\prime}$ where $X^{\prime}$ is a subcomplex of a $C W$ complex $Y^{\prime}$.

(ii) $E_{f}$ and $C_{f}$ also belong to $\mathfrak{W}$.

Proof of Lemma. By using the mapping cylinder argument we may assume that $f$ is an inclusion. Then we have a commutative diagram

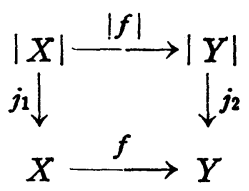

where $|X|$ and $|Y|$ are respectively the geometric realizations (see [4]) of the singular complexes of $X, Y$ and $|f|$ is induced by $f . j_{1}$ and $j_{2}$ are canonical maps which induce isomorphisms of homotopy groups [4, Theorem 4], and hence homotopy equivalences by [8, Theorem 1]. Taking $X^{\prime}, Y^{\prime}$ to be $|X|$, $|Y|$, we obtain the first assertion. We shall now prove the second half. In view of $[6$, Lemmas 6,9] and (i), we may assume that $X$ is a subcomplex of a $C W$-complex $Y$ and that $f$ is an inclusion. Obviously, $C_{f}=Y(/ C X$ is then 
a $C W$-complex. On the other hand, $E_{f}$ is the space of paths in $Y$ emanating from the base-point and ending in $X$. Milnor's result [5, Theorem 3] implies that $E_{f}$ belongs to $\mathfrak{B}$. This completes the proof of the lemma.

Proof of Theorem 2. By the preceding lemma, $\eta^{\prime}$ is equivalent to an inclusion $i: A \subset B$ where $A$ is a subcomplex of a $C W$-complex $B$. Hence, to prove our theorem, it suffices to show that $i$ induces $i^{*}: \pi(B, Z) \rightarrow \pi(A, Z)$ having the property stated in Theorem 2. Theorem 1 and the exactness of the cohomology sequence of the pair $(B, A)$ now imply that $H^{q}(B, A ; G)=0$ for $q \leqq r+s+2$ and for all coefficient groups $G$.

Firstly, let $\pi_{q}(Z)=0$ for $q \geqq r+s+2$; then $H^{q+1}\left(B, A ; \pi_{q}(Z)\right)=0$ for all $q \geqq 1$. Thus a standard obstruction argument shows that $i^{*}$ is onto. Secondly, suppose $\pi_{q}(Z)=0$ for $q \geqq r+s+3$; in this case $H^{q}\left(B, A ; \pi_{q}(Z)\right)$ vanishes for every $q \geqq 1$. Therefore, by the same reasoning, we see that $i^{*}$ is $1-1$.

Proof of Theorem 2'. Consider the mapping cylinder $M$ of $\eta: E_{f} \rightarrow \Omega C_{f}$. We have a homotopy-commutative diagram:

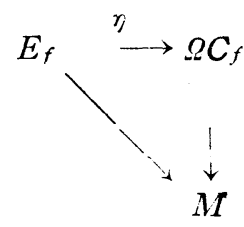

where the unlabelled arrows are inclusions and the vertical one is a homotopy equivalence. According to Theorem $1^{\prime}$, we have $\pi_{q}\left(M, E_{f}\right)=0$ for $1 \leqq q \leqq r+s$. Let $|Z|$ be the geometric realization of the singular complex of $Z$ and $j$ : $|Z| \rightarrow Z$ be the canonical map. In case $(\mathrm{a}), H^{q-1}\left(|Z| ; \pi_{q}\left(M, E_{f}\right)\right)=0$ for all $q \geqq 2$, and, in case (b), $H^{q}\left(|Z| ; \pi_{q}\left(M, E_{f}\right)\right)=0$ for all $q \geqq 2$. Observe also that our assumption implies $\pi_{1}\left(E_{f}\right)=0$ and hence the pair $\left(M, E_{f}\right)$ is $n$-simple for every $n \geqq 2$. Noting that $j$ is an equivalence and using the theory of obstructions to compressions as outlined in [2, Theorem 4.4.2], we obtain the desired conclusions.

Corollary 3. (J. Stasheff [7, Theorem 2]) Suppose $Y$ is a r-connected space belonging to 2 , and that $Z$ satisfies the condition $\pi_{q}(Z)=0$ for $q \geqq 2 r+1$ $(r \geqq 1)$. Then $\pi(Y, Z)$ is in $1-1$ correspondence with $\pi(\Omega Y, \Omega Z)$.

Proof. Taking $X$ to be a point in Theorem ?, we have $E_{f}=\Omega Y, C_{f}=Y$, 
and $s=r-1$. The assertion then follows at once. Note that $\sigma^{*}: \pi(Y, Z)$ $\rightarrow \pi(S \Omega Y, Z) \approx \pi(\Omega Y, \Omega Z)$ is seen to be the mapping induced by assigning to $v: Y \rightarrow Z \Omega v: \Omega Y \rightarrow \Omega Z$.

Similarly, by taking $Y$ to be a point in Theorem $2^{\prime}$, we have

Corollary $3^{\prime}$. Let $X$ be r-connected and let $Z$ be a $C W$-complex with $\operatorname{dim} Z \leqq 2 r$. Then $\pi(Z, X)$ is in $1-1$ correspondence with $\pi(S Z, S X)$.

\section{§. Applications}

First, the notations introduced in [6] will be used throughout; in particular, we consider $\tilde{\sigma}^{*}: \pi\left(C_{f}, Z\right) \rightarrow \pi\left(E_{f}, \Omega Z\right)$ given by $\bar{\sigma}^{*}\{v\}=\{\eta f, u\}$, where $v: C_{f} \rightarrow Z, u=v \circ P^{\prime} f: Y \rightarrow C_{f} \rightarrow Z$, and $\eta_{f, u}: E_{f \rightarrow \Omega} Z$ is defined by

$$
\left\{\eta_{f, u}(x, \beta)\right\}(s)= \begin{cases}v(x, 2 s) & \text { if } 0 \leqq s \leqq \frac{1}{2} \\ u \beta(2-2 s) & \text { if } \frac{1}{2} \leqq s \leqq 1\end{cases}
$$

Note that $\vec{\sigma}^{*}$ corresponds to $-\sigma^{*}$ (see [6, Lemma 14]).

Next, let $F \stackrel{i}{\rightarrow} X \stackrel{f}{\longrightarrow} Y$ and $F^{\prime} \stackrel{i^{\prime}}{\longrightarrow} X^{\prime} \stackrel{f^{\prime}}{\rightarrow} Y^{\prime}$ be two fiberings with fibres $F$, $F^{\prime}$ respectively, and let the following diagram be commutative:

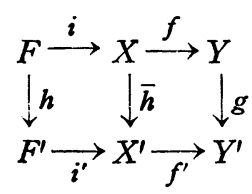

If $g, \bar{h}, h$ are all (weak) homotopy equivalences, we say that $(h, \bar{h}, g): f \rightarrow f^{\prime}$ is a (weak) equivalence between two fiberings.

Finally, we define $\{Y, Z\}$ to be the set of equivalence classes of elements in $\pi(Y, Z)$, in which we call $u_{1}, u_{2}: Y \rightarrow Z$ equivalent if and only if there exist homotopy equivalences $h: Z \rightarrow Z, k: Y \rightarrow Y$ satisfying $u_{2} \simeq h u_{1} k$.

Under these definitions, the main result obtained by applying Theorem 2 is stated as follows:

THEOREM 4. Let $Y$ and $Z$ belong to $\mathfrak{W}$, and let $Y$ be $(r-1)$-connected $(r \geqq 2)$. Suppose further that $\pi_{q}(Z) \neq 0$ onlv for $s+1 \leqq q \leqq r+s-1 \quad(s \geqq 1)$ and that $s+1 \geqq r$. Then the cquivalence classes of fiberings in $\mathfrak{W}$, uth $Y$ as base and with fibre $2 Z$, are in $1-1$ correspondence with $\{Y, Z\}$.

We list an immediate consequence of this theorem which seems to be well 
known :

Corollary. Let $Y$ be a 1-connected space in 2 , and let $Z$ be a space of type $(\pi, n+1)$ which also belongs to $\mathfrak{B}$. Then the classes of fiberings in $\mathfrak{B}$ having $Y$ as base and $\Omega Z$ as fibre are in $1-1$ correspondence with the equivalence classes in $H^{n+1}(Y, \pi)$ under $\Theta$, where $\Theta$ is the group of automorphisms of $H^{n+1}(Y, \pi)$ determined by homotopy equivalences of $Y$ and automorphisms of $\pi$.

The $1-1$ correspondence in Theorem 4 is established by assigning to $u: Y \rightarrow Z$ the class of the fibering $P u: E_{u} \rightarrow Y$ induced by $u$ from $E Z \rightarrow Z$. The fact that this is really $1-1$ will be readily seen by combining several lemmas below.

The following result is due to T. Ganea [3, Lemma 2.1]:

Lemma 1. Let $F \stackrel{i}{\longrightarrow} X \stackrel{f}{\longrightarrow} Y$ be a fibering with $X$ and $Y$ both in $\mathfrak{B}$. Suppose $Y$ is $(r-1)$-connected and that $\pi_{q}(F) \neq 0$ only for $s \leqq q \leqq r+s-2(r \geqq 2, s \geqq 1)$. Suppose further that there exists a weak equivalence $\theta: F \rightarrow \Omega Z$, where $Z$ is a 1-connected space. Then we can find a map $u: Y \rightarrow Z$ such that there exists a veak equivalence $(\xi, \bar{\xi}, 1): f \rightarrow P u$ with $\xi \simeq \theta$.

Proof. By assumption, $\pi_{q}(Z) \approx \pi_{q-1}(\Omega Z) \approx \pi_{q-1}(F)=0$ for $q \geqq r+s$. Further, since $F$ and $E_{f}$ are equivalent to each other (see [6, Theorem 1$]$ ), $E_{f}$ is $(s-1)$ connected. Thus Theorem 2, (a) implies that $\bar{\sigma}^{*}: \pi\left(C_{f}, Z\right) \rightarrow \pi\left(E_{f}, \Omega Z\right)$ is onto, i.e. there exists a map $v: C_{f} \rightarrow Z$ such that $\eta_{f, u} \simeq \theta \Psi$ where $u$ is the composition $Y \stackrel{P^{\prime} f}{\rightarrow} C_{f} \stackrel{v}{\rightarrow} Z$ and $\Psi: E_{f} \rightarrow F$ the canonical equivalence. We have a homotopy-commutative diagram:

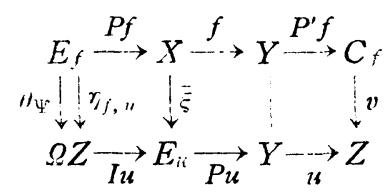

in which the middle square is commutative, and so $\overline{\bar{\xi}}$ induces $\hat{\xi}: F \rightarrow \Omega Z$. Applying the five lemma to the diagram of homotopy groups derived from the above one, we conclude that is a weak equivalence. Since a simple computation shows that 


$$
\begin{aligned}
& \xi(x)(s)=v(x, s) \\
& \text { for } x \in F, 0 \leqq s \leqq 1 \text {, } \\
& \left\{\eta f, u^{\circ} \Phi(x)\right\}(s)= \begin{cases}v(x, 2 s) & \text { for } 0 \leqq s \leqq \frac{1}{2}, \\
z_{0}=\text { base-point of } Z & \text { for } \frac{1}{2} \leqq s \leqq 1,\end{cases}
\end{aligned}
$$

where $\Phi: F \rightarrow E_{f}$ is a canonical inverse of $\Psi$ given by $\Phi(x)=(x, e), e$ being the constant path at $y_{0}$, we have $\theta \simeq \xi$, as asserted.

Next, in order to examine the extent to which $u$ is determined, we need

LeMma 2. Let $h: Z \rightarrow Z^{\prime}$ be a map; then $\sigma^{*} h_{*}=h_{*} \sigma^{*}, \bar{\sigma}^{*} h_{*}=(\Omega h)_{*} \bar{\sigma}^{*}$.

LeMma 3. In Lemma 1 we assume moreover that $Z$ belongs to $\mathfrak{B}$ and that $s+1 \geqq r$ (this is always the case when $r=2$ ). Suppose there is given the commutative diagram

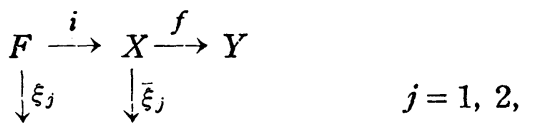

$$
\begin{aligned}
& \Omega Z \underset{I u_{j}}{\longrightarrow} E_{u_{j}} \underset{P u_{j}}{\longrightarrow} Y \underset{u_{j}}{\longrightarrow} Z
\end{aligned}
$$

in which vertical maps are (weak) equivalences. Then there exists an equivalence $h: Z \rightarrow Z$ such that $u_{2} \simeq h u_{1}$ and $\xi_{2} \simeq \Omega h \circ \xi_{1}$.

Proof. Let $\widetilde{\xi}_{1}$ be a homotopy inverse of $\xi_{1}$; since $Z$ is $s$-connected and $r+s \leqq 2 s+1$, we can apply Corollary 3 to obtain a map $h: Z \rightarrow Z$ such that $\Omega h \simeq \xi_{2} \circ \widetilde{亏}_{1}: \Omega Z \rightarrow \Omega Z$. Consider $v_{j}: C_{f} \rightarrow Z$ given by $(j=1,2)$

$$
\begin{array}{clrl}
v_{j}(y) & =u_{j}(y) & & \text { for } y \in Y, \\
v_{j}(x, s) & =\gamma_{j}(x)(s) & & \text { for } x \in X, 0 \leqq s \leqq 1,
\end{array}
$$

where $\bar{\xi}_{j}(x)=\left(f(x), \gamma_{j}(x)\right)$. Then it follows from the proof of Lemma 1 that $\mathscr{D}^{*} \vec{\sigma}^{*}\left\{v_{j}\right\}=\left\{\xi_{j}\right\}$. This leads to the following :

$$
\begin{aligned}
\mathscr{D}^{*} \bar{\sigma}^{*} h_{*}\left\{v_{1}\right\} & =\Phi^{*}(\Omega h)_{*} \bar{\sigma}^{*}\left\{v_{1}\right\} \quad \text { by Lemma } 2, \\
& =(\Omega h)_{*} \Phi^{*} \bar{\sigma}^{*}\left\{v_{1}\right\}=(\Omega h)_{*}\left\{\xi_{1}\right\} \\
& =\left\{\xi_{2}\right\}=\mathscr{D}^{*} \bar{\sigma}^{*}\left\{v_{2}\right\} .
\end{aligned}
$$

Because $\bar{\sigma}^{*}$ is $1-1$ by Theorem 2, (b) and $\Phi^{*}$ is an isomorphism, we have $\left\{h v_{1}\right\}=\left\{v_{2}\right\}$, whence, composing $P^{\prime} f$ to the right, $\left\{h u_{1}\right\}=\left\{\boldsymbol{u}_{2}\right\}$.

Lemma 4. Let $u: Y \rightarrow Z$ be a map and let $h: Z \rightarrow Z^{\prime}, k: Y^{\prime} \rightarrow Y$ be homotopy equivalences. Then $P u: E_{l} \rightarrow Y$ and $P(h u k): E_{h u k} \rightarrow Y^{\prime}$ are equivalent. 
Proof. Let $\widetilde{k}$ be a homotopy inverse of $k$. Consider then the following homotopy-commutative diagram

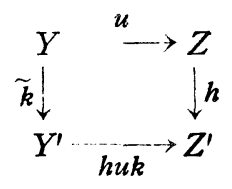

This transformation gives rise to the map $\%: E_{u} \rightarrow E_{h u k}$ which is defined by

$$
\varkappa(y, \gamma)=\left(\widetilde{k}(y), \gamma^{\prime}(y)\right) \quad \text { for } y \in Y, \gamma \in E Z, u(y)=\gamma(1),
$$

where $\gamma^{\prime}(y)$ is the path in $Z^{\prime}$ given by, using a homotopy $H_{t}: Y \rightarrow Z^{\prime}$ with $H_{0}=h u, H_{1}=h u k \widetilde{k}, \gamma^{\prime}(y)(s)=h \gamma(2 s)$ for $0 \leqq s \leqq \frac{1}{2},=H_{2 s-1}(y)$ for $\frac{1}{2} \leqq s \leqq 1$. It follows from Lemma 6 in [6] that $\%$ is a homotopy equivalence. Thus $(\Omega h, \%, \widetilde{k}): P u \rightarrow P(h u k)$ is a desired equivalence.

Lemma 5. Suppose there is given the commutative diagram

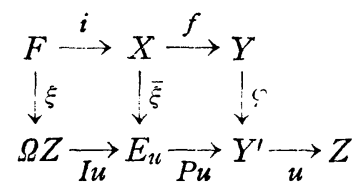

where the top line is a fibering. Then the above diagram admits a factorization:

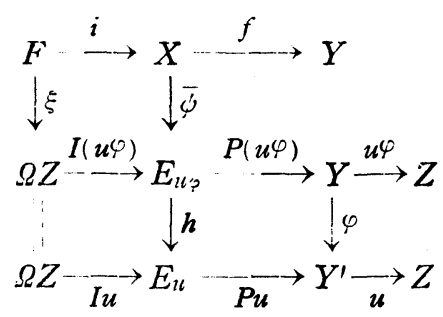

Proof. If we write down $\overline{\bar{\xi}}$ as $\bar{\xi}(x)=(\varphi f(x), \gamma(x))$ for $x \in X, \gamma(x) \in E Z$ with $u \varphi f(x)=\gamma(x)(1)$, then it suffices to consider $\bar{\psi}, h$ given by $\bar{\psi}(x)=(f(x)$, $\gamma(x))$, and $h(y, \gamma)=(\varphi(y), \gamma), y \in Y, \gamma \in E Z$.

Finally, we shall prove

THEOREM 5. Let $f: \Omega W \rightarrow \Omega V$ be a map with $W, V$ in $\mathfrak{W}$. Suppose further that $\pi_{i}(W) \neq 0$ only for $n+1 \leqq i \leqq 2 n$ and $\pi_{j}(V) \neq 0$ only for $q+2 \leqq j \leqq n+q+1$ $(2 \leqq n \leqq q+1)$. If $E_{f}$ is of the same homotopy type as a loop-space of a space in $\mathfrak{B}$, then $f$ is homotopic to $\Omega g$ for some $g: W \rightarrow V$. 
Proof. Let $\theta: E_{f} \rightarrow \Omega Z$ be an equivalence. For $q=n-1$, the assertion is trivial by Corollary 3. Hence we may assume that $q \geqq n$. Since $E_{f}$ is $(n-1)$ connected and $\pi_{i}(Z)=0$ for $i \geqq n+q+1$, we can apply Theorem 2, (a) to find a map $v: C_{f} \rightarrow Z$ such that there exists a homotopy-commutative diagram

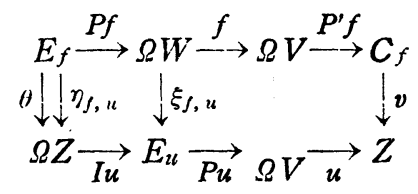

in which $\theta \simeq \eta_{f, u}, u=v \circ P^{\prime} f$. By the five lemma we see that $\xi_{f, u}$ is a homotopy equivalence. Choose a homotopy inverse $\rho: E_{u} \rightarrow \Omega W$ of $\xi_{f, u}$. Observe that $Z$ and $E_{u}$ are $n$ - and $(n-1)$-connected respectively. Thus Theorem 2 yields again a map $v^{\prime}: C_{n} \rightarrow W$ such that $\eta_{u, w} \simeq \rho$ holds in the homotopycommutative diagram

$$
\begin{gathered}
E_{u} \stackrel{P u}{\longrightarrow} \Omega V \stackrel{u}{\rightarrow} Z \stackrel{P^{\prime} u}{\rightarrow} C_{u} \\
o \downarrow \downarrow \eta_{u, w} \downarrow^{\prime} \xi_{u, w} \\
\Omega W \underset{I w}{\longrightarrow} E_{w} \underset{P w}{\longrightarrow} Z \underset{w}{\rightarrow} W
\end{gathered}
$$

where $w=v^{\prime} \circ P^{\prime} u$. As above, $\xi_{u, w}$ is seen to be an equivalence. Let $\tau$ : $E_{w} \rightarrow \Omega V$ be a homotopy inverse of $\xi_{u, w}$. Since $E_{w}$ is $q$-connected, Theorem 2 shows that there exists a homotopy-commutative diagram

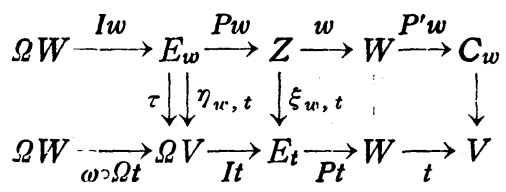

where $\tau \simeq \eta w, t$ and $\omega$ is the involution induced by reversing loop-parameter. Combining the above three diagrams with one another we see that $f \simeq \omega \circ \Omega t$. Corollary 3 implies that $\omega \simeq \Omega s$ for some $s: V \rightarrow V$, whence $f \simeq \Omega g$ for $g=s \circ t$.

Remark. By a similar argument as in Theorem 5 we may obtain a result due to Berstein and Hilton ([1, Lemma 3.6]): Let $f: S^{m-1} \rightarrow S^{q}, m \geqq q+1 \geqq 3$. If $C_{f}=S^{a} \bigcup_{f} e^{m}$ is of the same homotopy type as a suspension, then $f$ is homotopic to Sg for some $g: S^{m-2} \rightarrow S^{q-1}$.

Added in proof. After the submission of this note, Hilton's paper "On excision and principal fibrations" (Comment. Math. Helv. 35, 1961, Fasc. 2) 
appeared, where he has obtained essentially the same results as our Theorems 2 and $2^{\prime}$.

\section{REFERENCES}

[1] I. Berstein and P. J. Hilton, Category and generalized Hopf invariants, Illinois J. of Math. 4 (1960), 437-451.

[2] A. L. Blakers and W. S. Massey, The homotopy groups of a triad I, Ann. of Math. 33 (1951), 161-205.

[3] T. Ganea, Fibrations and Cocategory, Comment. Math. Helv. 35 (1961), 15-24.

[4] J. Milnor, The geometric realization of a semi-simplicial complex, Ann. of Math. 65 (1957), 357-362.

[5] J. Milnor, On spaces having the homotopy type of a $C W$-complex, Trans. Amer. Math. Soc. 90 (1957), 272-280.

[6] Y. Nomura, On mapping sequences, Nagoya Math. J. 17 (1960), 111-145.

[7] J. Stasheff, On the space-of-loops isomorphism, Proc. Amer. Math. Soc. 10 (1959), 987-993.

[8] J. H. C. Whitehead, Combinatorial homotopy I, Bull. Amer. Math. Soc. 55 (1949), 245-277.

Department of Mathematics

Shizuoka University

Shizuoka, Japan 